Universidade Tecnológica Federal do Paraná - UTFPR

Campus Ponta Grossa - Paraná - Brasil

ISSN: $1981-3686 /$ v. 05 , n. $02:$ p. $573-583,2011$

D.O.I.: $10.3895 / \mathrm{S} 1981-36862011000200009$

\section{Revista Brasileira de Tecnologia Agroindustrial}

\title{
CARACTERIZAÇÃO FÍSICA, FÍSICO-QUÍMICA E POTENCIAL TECNOLÓGICO DE FRUTOS DE CAMAPU(Physalis angulata L.)
}

\section{PHYSICAL, PHYSICO-CHEMICAL AND TECHNOLOGICAL POTENTIAL OF CAMAPU FRUITS (Physalis angulata L.)}

\author{
Johnatt Allan Rocha De Oliveira ${ }^{1}$, Luiza Helena da Silva Martins ${ }^{2}$, Marcus Arthur Marçal de Vasconcelos ${ }^{3}$, \\ Rosinelson da Silva Pena ${ }^{4}$, Ana Vânia Carvalho ${ }^{5}$ \\ ${ }^{1,2}$ UNICAMP, Campinas, Brasil johnattrocha@yahoo.com.br; luizahelena84@yahoo.com.br \\ ${ }^{3,5}$ EMBRAPA Amazônia Oriental mavasc@cpatu.embrapa.br \\ ${ }^{4}$ Universidade Federal do Pará (UFPA) rspena@ ufpa.br; anavania@ cpatu.embrapa.br
}

\begin{abstract}
RESUMO
O objetivo deste estudo foi investigar o potencial tecnólogico de frutos de camapu. Para isso foram determinadas as características físicas, físico-químicas e os teores de vitamina $C$, carotenóides totais, compostos fenólicos totais e a capacidade de seqüestrar radicais livres através do método DPPH (2,2-difenil-1-picril hidrazil). Alguns dos resultados observados foram: sólidos solúveis de $12^{\circ}$ Brix; $\mathrm{pH}$ de 4,11; Aw de 0,97; teor de proteínas de 0,95\%; 6,45\% de açúcares totais e 4,12\% de açúcares redutores; $25 \mathrm{mg} .100 \mathrm{~g}^{-1}$ de vitamina $C$; 3,99 $\mu \mathrm{g} \mathrm{g}^{-1}$ de carotenoides totais; $61,70 \mathrm{mg}$ GAE. $100 \mathrm{~g}^{-1}$ para compostos fenólicos totais e a capacidade antioxidante de IC $_{50}$ : 93,01 e 92,8 $\mu g . m L^{-1}$ para extração aquosa e metanólica respectivamente. Os resultados obtidos mostraram teores consideráveis de fenólicos totais e atividade antioxidante mediana para os frutos de camapu, comparáveis a outras frutas já amplamente comercializadas no Brasil.
\end{abstract}

Palavras-chave: antioxidantes, carotenos, Amazônia, agroidústria

\section{INTRODUÇÃO}

As características físicas e químicas dos frutos são de grande importância para a sua comercialização e manuseio. A aparência externa dos frutos, tais como tamanho, consistência, espessura, forma e coloração da casca são fatores importantes para a aceitabilidade pelos consumidores (COSTA et al., 2004).

Nesse mercado, embora o Brasil seja destaque na produção de diferentes variedades frutíferas nativas ou exóticas (GRANADA et al., 2004), acredita-se que a fruticultura nacional tenha ainda grande potencial de expansão, pois há inúmeras frutas nativas e exóticas pouco exploradas economicamente. Estudos para transformá-las em culturas racionais, na sua maioria, estão em andamento, como por exemplo, atemóia, maná, canistel, mirtilo, lichia, physalis, carambola, entre outras (LAGO et al., 2006). 
O camapu (Physalis angulata L.) pertence à família Solanaceae a qual está distribuída ao longo de todas as regiões tropicais e subtropicais do mundo (KISSMANN e GROTH, 1995). No Brasil, Physalis angulata é conhecido popularmente como "Camapu", "Bucho de Rã", "Juá de Capote" ou "Mata-Fome" (FREITAS et al., 2006), e seu suco é considerado como sedativo depurativo e contra o reumatismo (LORENZI, 1982).

Os frutos de camapu contêm, além dos nutrientes essenciais e de micronutrientes como minerais, fibras, vitaminas, diversos compostos secundários de natureza fenólica, denominados compostos fenólicos (HARBONE e WILLIAMS, 2000). Estudos clínicos e epidemiológicos têm mostrado evidências de que antioxidantes fenólicos de cereais, frutas e vegetais são os principais fatores que contribuem para a baixa e significativa redução da incidência de doenças crônicas e degenerativas encontradas em populações cujas dietas se caracterizam por uma elevada ingestão desses alimentos (SHAHIDI, 2008).

Poucos estudos foram realizados com base na caracterização dos frutos de camapu. Dessa forma o presente trabalho objetivou o estudo das características físicas, físico-químicas e potencial tecnológico de frutos inteiros de camapu (Physalis angulata) (semente, casca e polpa) em estádio "de vez".

\section{MATERIAL E MÉTODOS}

\section{Material}

Três amostras totalizando 500g de frutos de Physalis Angulata L., safra 2007 e 2008, com ocorrência natural na área da Embrapa Amazônia Oriental, foram colhidas no estádio "de vez", e imediatamente tranportadas até o Laboratório de Agroindústria da Embrapa Amazônia Oriental. Para todas as análises os frutos foram submetidos a um banho térmico de $80{ }^{\circ} \mathrm{C}$ durante 5 minutos, seguido por outro banho a $0{ }^{\circ} \mathrm{C}$. Uma parte desses frutos seguiu para as extrações e análises de DPPH e outra foi triturada e congelada a $-80{ }^{\circ} \mathrm{C}$ para a caracterização físico-química e possíveis repetições de análises.

\section{Métodos}

\section{Caracterização física dos frutos in natura}

Utilizou-se 100 unidades de frutos, escolhidos aleatoriamente. Os frutos foram pesados em balança eletrônica da marca GEHAKA, modelo BG-4000 e suas dimensões, comprimento e largura, 
foram medidas com auxílio de um paquímetro metálico da marca VONDER, com escala de 300 $\mathrm{mm}$.

\section{Caracterização físico-química}

No material termicamente tratado e triturado foram determinados: $\mathrm{pH}$ (AOAC, 1997), acidez titulável (AOAC, 1997), sólidos solúveis (AOAC, 1997), atividade de água (medição direta em analisador de atividade de água da marca DECAGON®, modelo Pawkit, Pullman, EUA), umidade (AOAC, 1997), cinzas (AOAC, 1997), proteínas (AOAC, 1997), lipídios (BLIGH e DYER, 1959), fibras (GOERING e VAN SOEST, 1970), açúcares totais e redutores (LANE \& EYNON), vitamina C (segundo método n ${ }^{\circ} 43.065$ da AOAC, 1984, modificado por Benassi, 1990) e carotenoides totais (RODRIGUEZ-AMAYA, 1999).

\section{Extração e determinação de compostos fenólicos totais}

A extração dos compostos fenólicos foi realizada com solventes em procedimento seqüencial de acordo com literaturas específicas (SILVA et al., 2007; ROESLER, 2007) em balões âmbar de 30mL, usando uma razão sólido-líquido de 1:20 (m:v) (1g de amostra : $20 \mathrm{~mL}$ da primeira solução extratora) com três diferentes soluções extratoras. As soluções extratoras utilizadas foram: primeira extração - acetona: metanol (70:30, v:v); segunda extração - metanol:água (70:30, v:v) e a terceira extração - metanol:água:ácido acético (50:40:10, v:v:v).

Em um balão âmbar foram depositadas as amostras do fruto seco em pó e em seguida adicionados o volume da primeira solução extratora. $\mathrm{O}$ balão ficou em repouso por 45 minutos a 58 ${ }^{\circ} \mathrm{C}$. Em seguida a solução foi filtrada em kitassato de $100 \mathrm{~mL}$ com auxílio de uma bomba de vácuo. O material retido no papel de filtro foi coletado e transferido para outro balão âmbar, tomando-se cuidado para que o papel filtro fosse lavado com a segunda solução extratora $(20 \mathrm{~mL})$, repetindo-se os procedimentos para a terceira solução extratora como já mencionados para as duas primeiras. $\mathrm{O}$ extrato resultante das três lavagens foi coletado no kitassato e em seguida misturado e analisado.

A concentração dos polifenóis foi determinada pelo método colorimétrico de FolinCiocalteau (SINGLETON et al., 1999) adaptado por Silva et al. (2007) para uso em microplacas.

A partir de cada extrato, foram preparadas em triplicata soluções estoque pelos seguintes procedimentos: $5 \mathrm{mg}$ de extrato foram solubilizados com $1 \mathrm{~mL}$ de metanol, sonificados por 3 minutos em banho ultrasônico, sendo em seguida centrifugados por 5 minutos a $3500 \mathrm{rpm}$ e $4{ }^{\circ} \mathrm{C}$. O sobrenadante foi transferido para tubos do tipo Eppendorf de $2 \mathrm{~mL}$, envoltos por papel alumínio e saturados com $\mathrm{N}_{2}$. Estas soluções eram armazenadas a $4{ }^{\circ} \mathrm{C}$, por até dois dias.

Em seguida teve início a mistura reacional realizada com $250 \mu \mathrm{L}$ de extrato, $125 \mu \mathrm{L}$ de solução de Folin-Cioucateu $1 \mathrm{~N}$ e $625 \mu \mathrm{L}$ de solução aquosa de carbonato de sódio $\left(\mathrm{Na}_{2} \mathrm{CO}_{3}\right)$ a 
$75 \mathrm{~g} / \mathrm{L}$. Após o período de incubação de 30 minutos à temperatura ambiente $\left(25^{\circ} \mathrm{C}\right), 200 \mu \mathrm{L}$ da mistura reacional foram transferidos para os poços da microplaca (transparentes) e a absorbância lida em espectrofotômetro (Ultospec 2000, UV visível - Pharmacia Biotech, EUA) a 765 nm.

O teor de compostos fenólicos totais foi calculado a partir da curva de calibração do ácido gálico e expresso como miligramas de equivalentes de ácido gálico por grama de extrato seco (mgEAG/gES). Todas as leituras foram feitas em triplicatas sendo refeitas aquelas que apresentarem um erro relativo maior ou igual a $12 \%$.

\section{Extração aquosa para DPPH}

Os frutos inteiros e termicamente tratados foram triturados em liquidificador por aproximadamente 5 minutos com água destilada na proporção de $1: 3\left(\mathrm{~m} . \mathrm{m}^{-1}\right)$ fruta: água. O material foi filtrado em gazes e o resíduo foi submetido à nova extração com água nas mesmas condições. $\mathrm{O}$ material filtrado, bem como o material retido no filtro, foi liofilizado a $-18{ }^{\circ} \mathrm{C}$ a $13,3 \mathrm{~Pa}$. Os extratos liofilizados foram armazenados em frascos âmbar a $-18^{\circ} \mathrm{C}$ até o momento das análises.

\section{Extração etanólica para DPPH}

Os frutos inteiros foram triturados em liquidificador por aproximadamente 5 minutos sem adição de água, em seguida macerados em almofariz e a massa resultante foi utilizada para a extração. A extração foi realizada com solução aquosa de etanol (5:95, v.v ${ }^{-1}$, água:etanol) na proporção de 1:3 (m.m $\left.\mathrm{m}^{-1}\right)$ fruta: solução de etanol. O material foi filtrado em gaze e o resíduo foi reextraído nas mesmas condições. Os materiais obtidos, resíduo e extrato etanólico, foram concentrados em rota-evaporador a $40{ }^{\circ} \mathrm{C}$, sendo que o extrato concentrado e os resíduos obtidos foram liofilizados a $-18{ }^{\circ} \mathrm{C}$ a $13,3 \mathrm{~Pa}$ e armazenados em frascos âmbar a $-18{ }^{\circ} \mathrm{C}$ até sua utilização.

\section{Determinação da capacidade de seqüestrar radicais livres}

A capacidade antioxidante foi avaliada utilizando-se o método do seqüestro de radicais livres do DPPH (2,2 difenil-1-picrilhidrazil), que se baseia em um ensaio fotométrico onde o radical livre DPPH, que apresenta coloração roxa intensa em solução alcoólica, se reduz em presença de moléculas antioxidantes, formando o 2,2 difenil-1-picrilhidrazil, que é incolor. O método utilizado foi aquele descrito por Chang et al. (2006).

A partir dos extratos aquosos e etanólicos, soluções etanólicas com diferentes concentrações foram preparadas pela adição de $1000 \mathrm{~mL}$ de DPPH (0,004\% m.v-1), e o volume final foi ajustado para $1200 \mathrm{~mL}$ com etanol. A concentração final dos extratos nas cubetas foi de 1 $\mathrm{mg} \mathrm{mL}^{-1}$ a $2000 \mathrm{mg} \mathrm{mL}^{-1}$. As amostras foram incubadas durante 30 minutos à temperatura ambiente no escuro. Para efeito comparativo o mesmo procedimento foi adotado para o ácido gálico e para o 
extrato comercial de alecrim. O controle foi preparado conforme procedimento acima, sem adição de extrato, e etanol foi utilizado para correção da linha de base. A solução de DPPH' foi preparada no mesmo dia, de maneira prévia a realização da análise e estocada em frascos cobertos com folhas de alumínio, sob proteção da luz $4{ }^{\circ} \mathrm{C}$ até o momento das determinações. O percentual de decréscimo na absorbância foi medido para cada concentração e a capacidade de seqüestrar radicais livres foi calculada com base no decréscimo da absorbância observada. A absorbância das amostras foram realizadas a $517 \mathrm{~nm}$. A capacidade de seqüestrar radical livre foi expressa como percentual de inibição de oxidação do radical e calculado conforme fórmula abaixo (YEN e DUH, 1994):

$$
\% \text { Inibição }=((\mathrm{ADPPH}-\mathrm{AExtr}) / \mathrm{ADPPH}) * 100
$$

Onde:

ADPPH é a absorbância da solução de DPPH';

AExtr é a absorbância da amostra em solução.

AExtr foi calculado com base na diferença da absorbância da solução de amostra em teste com seu branco. $\mathrm{O}$ valor de $\mathrm{IC}_{50}$ é definido com a concentração final em $\mathrm{mg} \mathrm{mL}^{-1}$ do extrato seco presente na cubeta, requerido para decrescer a concentração inicial de DPPH em 50\%.

\section{Análise Estatística}

Os resultados foram submetidos à análise de variância, e as médias, quando significativas, comparadas pelo Teste de Tukey a $5 \%$ de significância, com auxílio do programa SAS 8.0 (Statistical Analysis System).

\section{RESULTADOS E DISCUSSÃO}

\section{Caracterização física}

Os resultados da caracterização física do camapu estão apresentados na Tabela 1. Tabela 1 - Caracterização física do camapu

\begin{tabular}{llll}
\hline & Máximo & Mínimo & Médio* \\
\hline Peso $(\mathrm{g})$ & 7,06 & 1,50 & $4,33 \pm 2,107$ \\
Comprimento $(\mathrm{cm})$ & 2,40 & 1,38 & $2,17 \pm 0,325$ \\
Largura $(\mathrm{cm})$ & 2,03 & 1,20 & $1,86 \pm 0,265$ \\
\hline \multicolumn{1}{c}{ Média de 100 determinações } & &
\end{tabular}

Não foram encontrados dados que referenciem as medidas físicas de frutos de camapu dessa forma optou-se por compará-los com frutos de tamanhos relativamente semelhantes como é o caso da acerola. O camapu utilizado nas análises apresentou tamanho grande quando comparado com 
dados obtidos por Cavalcante (1991) (comprimento médio de $2 \mathrm{~cm}$ e largura de 1,75 cm), Mattietto e Pontes (1997) (comprimento médio de $1,98 \mathrm{~cm}$ e largura de 1,67cm) e Mattietto (1999) (comprimento médio de 1,33 cm e largura de 1,14cm) para frutos de acerola.

\section{Caracterização físico-química}

Os resultados da caracterização físico-química do camapu encontram-se na Tabela 2.

Tabela 2 - Caracterização físico-química do fruto de camapu

\begin{tabular}{|c|c|}
\hline Determinação & Valor Médio* \\
\hline$\overline{\mathrm{pH}}$ & $4,11 \pm 0,012$ \\
\hline Sólidos solúveis $\left({ }^{\circ} \mathrm{Brix}\right.$ a $20^{\circ} \mathrm{C}$ ) & $12,00 \pm 0,004$ \\
\hline Acidez total titulável (\% ácido cítrico) & $0,68 \pm 0,004$ \\
\hline Atividade de água (Aw) & $0,97 \pm 0,010$ \\
\hline Lipídeos totais $(\%)$ & $0,59 \pm 0,037$ \\
\hline Proteína total $(\%)$ & $0,85 \pm 0,050$ \\
\hline Umidade $(\%)$ & $90,98 \pm 0,280$ \\
\hline Cinzas $(\%)$ & $0,65 \pm 0,022$ \\
\hline Fibras $(\%)$ & $0,84 \pm 0,040$ \\
\hline Açúcares totais $(\%)$ & $6,45 \pm 0,006$ \\
\hline Açúcares redutores $(\%)$ & $4,12 \pm 0,050$ \\
\hline Vitamina C (mg de ácido ascórbico/100g) & $25,00 \pm 0,461$ \\
\hline Carotenóides totais $\left(\mu \mathrm{g} \mathrm{g}^{-1}\right)$ calculado como $\beta$-caroteno & $3,99 \pm 0,030$ \\
\hline Compostos fenólicos (mg de GAE.100g ${ }^{-1}$ ) & $63,70 \pm 0,337$ \\
\hline
\end{tabular}

Há poucos dados referenciados em artigos científicos sobre as características físicoquímicas do camapu, o que ressalta a importância de novos estudos de caracterização para que a comercialização e exploração do potencial tecnológico do fruto possam ser alcançadas. Assim optou-se por comparar os resultados de caracterização com frutas já amplamente comercializadas no mercado brasileiro de frutas.

$\mathrm{O}$ valor de $\mathrm{pH}(4,11)$ encontrado é semelhante àqueles citados para goiabas por Gouveia et al. (2004) (3,9) e está próximo aos valores de 3,8 e 3,75 encontrados por Sarzi e Durigan (2002) para abacaxis da variedade Pérola. Para a acidez o valor encontrado $(0,68 \%)$ encontra-se dentro do intervalo citado por Argenta et al. (1995), para frutos de goiabeira (0,40 a 1,04\%), sendo maiores que os observados por Cantillano (1988) $(0,23 \%$ - 0,26\%) para maçãs. Em relação aos sólidos solúveis o valor encontrado para o camapu foi de $12^{\circ}$ Brix, semelhantes a outros frutos como: maçã (14 - 15,8 ${ }^{\circ}$ Brix) (CANTILLANO, 1988); goiaba (11 - 12,1 ${ }^{\circ}$ Brix) (MAIA et al., 1998) e abacaxi (9 - $13{ }^{\circ}$ Brix) (REINHARDT et al., 2004). O teor de sólidos solúveis totais é um índice de qualidade, sendo sua concentração e composição componente indispensável ao sabor do fruto. Outros parâmetros como pH e acidez titulável da polpa da fruta também são utilizados para indicar a qualidade dos frutos e refletem o estádio de maturação dos mesmos (SANTANA et al., 2004). 
Para o teor de açúcares totais e redutores foram encontrados os valores de 6,45 e 4,12\% respectivamente, bem diferente dos relatados para maçãs (10,35 e 1,21\%) (GOULARTE et al., 2000) e próximos aos relatados por Pantoja et al. (2009) que estudou frutos de tamarindo e encontrou valores de 7,62 e 5,28 para açúcares totais e redutores respectivamente.

Para o teor de proteína são relatados valores de 0,74 a 0,82\% (MAIA et al., 1998) e de 0,62 a 0,66\% (GOUVEIA et al., 2004) para diferentes variedades de goiaba. Estes valores se mostram inferiores aos encontrados para o camapu $(0,85 \%)$, semelhante ao que acontece quando estes são comparados com abacaxis para o qual Franco (1989) relata valor de 0,40 \%. Em relação ao teor de vitamina $\mathrm{C}$ obteve-se valor de $25 \mathrm{mg}$ de ácido ascórbico por $100 \mathrm{~g}$ de polpa, semelhante a outros frutos amplamente comercializados como: abacaxi $(28,72 \mathrm{mg}$ de ácido ascórbico/100g) (REINHARDT et al., 2004), morango (57,14 - 81,14 mg de ácido ascórbico/100g) (ROCHA et al., 2008), tangerina (21,50 mg de ácido ascórbico/100g) e laranja $(62,50$ - 84,03 mg de ácido ascórbico/100g) (COUTO e CANNIATTI-BRAZACA, 2010).

Para o teor de carotenóides totais foi encontrado o valor médio de $3,99 \mu \mathrm{g} \mathrm{g}^{-1}$, valor menor que aquele encontrado por Marinova e Ribarova (2006) para amora-preta $\left(4,4 \mu \mathrm{g} \mathrm{g}^{-1}\right)$. O mesmo autor obteve para a fruta de mirtilo um conteúdo de carotenóides totais de $2,9 \mu \mathrm{g} \mathrm{g}^{-1}$, valor inferior ao verificado no presente estudo. Lima et al. (2002) encontrou para pitangas valores de 79 a $111 \mu \mathrm{g}$ $\mathrm{g}^{-1}$, superiores aos observados para o camapu. Lima et al. (2005) determinaram o conteúdo de carotenóides totais em acerola em três estádios de maturação e encontraram valores variando entre 9,4 e 30,9 $\mu \mathrm{g} \mathrm{g}^{-1}$ (estação seca) e de 14,1 a 40,6 $\mu \mathrm{g} \mathrm{g}^{-1}$ (estação de chuvas), teores superiores ao observado para o camapu. De maneira geral, observa-se que o camapu não pode ser considerado uma fonte rica de carotenóides.

Os compostos fenólicos, presentes nas frutas e hortaliças, são um dos principais responsáveis pela atividade antioxidante destas. Seu conteúdo final pode ser influenciado por fatores como a maturação, a espécie, práticas de cultivo, origem geográfica, estádio de crescimento, condições de colheita e processo de armazenamento (KIM et al., 2003). Foi encontrado o teor de 63,70 mg de GAE.100 $\mathrm{g}^{-1}$ para os frutos de Physalis angulata, valor superior ao relatado por Rockenbach et al. (2008) para o extrato de Physalis peruviana que foi de 57,9 mg de GAE.100 g-1. O teor de fenólicos totais observado neste estudo é superior ao encontrado por Kuskoski et al. (2006) em polpa de abacaxi, cupuaçu e maracujá, que foram, respectivamente, de 21,7, 20,5 e 20,0 $\mathrm{mg}$ de GAE.100 $\mathrm{g}^{-1}$. No mesmo estudo, foram observados teores de fenólicos totais também inferiores para graviola (84,30 mg GAE. $\left.100 \mathrm{~g}^{-1}\right)$ e superiores para a uva $\left(117,1 \mathrm{mg}\right.$ de GAE. $100 \mathrm{~g}^{-}$ ${ }^{1}$ ), quando comparados aos teores observados no presente estudo. Sun et al. (2002) encontraram em frutas como uva (182,0 mg de GAE. $\left.100 \mathrm{~g}^{-1}\right)$ e morango (147,8 $\mathrm{mg}$ de GAE.100 g ${ }^{-1}$ ) valores também superiores aos apresentados para os de camapu. Já Wu et al. (2006) encontraram valores de 
compostos fenólicos totais em diferentes extratos de camapu (Physalis peruviana) variando entre 14,53 e $90,80 \mathrm{mg}$ de GAE. $100 \mathrm{~g}^{-1}$.

\title{
Capacidade antioxidante
}

A atividade antioxidante encontrada para o camapu (Physalis angulata) foi de $\mathrm{IC}_{50}=93,01$ $\mu \mathrm{g} \cdot \mathrm{mL}^{-1}$ para o extrato aquoso e de $\mathrm{IC}_{50}=92,8 \mu \mathrm{g} \mathrm{mL} \mathrm{m}^{-1}$ para o extrato metanólico, não sendo observada diferença significativa a 95\% de confiança. Os valores encontrados são superiores àqueles relatados por Kuskoski et al. (2005) para pinã, cupuaçu e maracujá que são de 41,10, 43,18, $46,66 \mu \mathrm{g} \mathrm{mL}^{-1}$, respectivamente. O mesmo autor cita valores para a manga, açaí, uva e goiaba (IC 50 $=174,30,108,50,105,90 \mathrm{e} 100,7 \mu \mathrm{g} \mathrm{mL}{ }^{-1}$ respectivamente) superiores aos encontrados para o camapu avaliado no presente estudo. Chang et al. (2008) encontraram valor de $\mathrm{IC}_{50}=0.89 \mu \mathrm{gL} \mathrm{m}^{-1}$ para camapus da variedade Physalis peruviana em extratos metanólicos semelhantes aos encontrados para o camapu da variedade Physalis angulata. Já Laczkó-Zöld et al. (2009) obteve valor de $\mathrm{IC}_{50}=22.32 \mu \mathrm{g} \mathrm{mL} \mathrm{m}^{-1}$ para camapus da variedade Physalis alkekengi também em extratos metanólicos, sendo este valor bem inferior ao relatado para o camapu avaliado neste trabalho.

\section{CONCLUSÃO}

De maneira geral, os frutos de camapu da variedade Physalis angulata analisados apresentaram teores significativos de vitamina $\mathrm{C}$ e atividade antioxidante mediana, o que constitui bom atrativo para o aproveitamento tecnológico dos frutos, ainda pouco comercializados na maior parte do Brasil.

\begin{abstract}
The aim of this study was to investigate the technological potential of the camapu fruits. For this we determine the physical, physical-chemical and the levels of vitamin $C$, carotenoids, phenolic compounds and the ability to scavenge free radicals by DPPH (2,2-diphenyl-1-picryl hydrazyl). Some results were: soluble solids of $12^{\circ}$ Brix, pH 4,11, Aw 0,97; protein content of $0.95 \%, 6.45 \%$ of

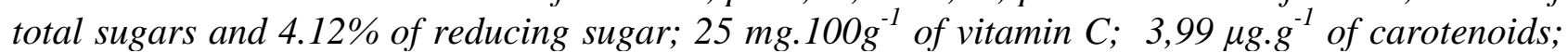
$61,70 \mathrm{mg} \mathrm{GAE} .100 \mathrm{~g}^{-1}$ for total phenolics and antioxidant capacity of $I_{50}: 93,1$ and 92,8 $\mu \mathrm{g} . \mathrm{mL}^{-1}$ for water extraction and methanol respectively. The results showed considerable concentrations of phenolics and antioxidant activity median for the fruits of Camapu, comparable to other fruit already widely sold in Brazil.
\end{abstract}

Key-words: antioxidant capacity, carotens, Amazon, agroindustry 


\section{REFERÊNCIAS}

AOAC. Official methods of analysis of the Association of Official Analytical Chemists: edited lg W. Horwitz 13 ed. Washington, 850p, 1984.

AOAC. Official methods of analysis of the Association of Official Analytical Chemists: edited $1 g$ W. Horwitz 16 ed. Washington, 850p, 1997.

ARGENTA, L. C.; BENDER, R. J.; KREUS, C. L.; MONDARDO, M. Padrões de maturação índices de colheita de maçãs CVS. Gala, Golden Delicious e Fuji. Pesquisa Agropecuária Brasileira, v. 30, n. 10, p. 1259-1166, 1995.

BENASSI, M. T. Análise dos efeitos de diferentes parâmetros de vitamina $\mathbf{C}$ em vegetais processados. Campinas, 1990. 159f. Dissertação (Mestre em Tecnologia de Alimentos) - Faculdade de Engenharia de Alimentos, Universidade Estadual de Campinas, 1990.

BLIGH, E. G.; DYER, W. J. A rapid method of total lipid extraction and purification. Canadian Journal of Biochemistry and Physiology, v. 37, n. 8, p. 911-917, 1959. http://dx.doi.org/10.1139/o59-099

CANTILLANO, R. F. F. Colheita da maçã: recomendações técnicas. Pelotas, RS: EMBRAPA-CNPFT (EMBRAPACNPFT. Comunicado Técnico, 62. 1988.

CAVAlCANTE, M. L. Composição de carotenóides e valor de vitamina A em pitanga (Eugenia uniflora) e acerola (Malphighia glabra L.). 1991. 87f. Dissertação (Mestrado em Ciência de Alimentos) - Faculdade de Engenharia de alimentos, Universidade Estadual de Campinas, Campinas, 1991.

CHANG, J.C. LIN, C. C.; WU, S. J.; LIN, D. L.; WANG, S. S.; MIAW, C. L.; NG, L. T. Antioxidative and Hepatoprotective Effects of Physalis peruviana Extract against Acetaminophen-Induced Liver Injury in Rats. Pharmaceutical Biology, v. 46, n. 10-11, p. 724-731. 2008. http://dx.doi.org/10.1080/13880200802215768

CHANG, W. C.; LEE, E. L.; NG, L. T. Factors influencing the 2,2- diphenyl-1-picrylhydrazyl (DPPH) radical scavenging assay in the natural product analysis. Tajen Journal, v. 28, n. 2, p. 17-36, 2006.

COSTA, N. P.; LUZ, T. L. B.; BRUNO, R. L. A. Caracterização físico-química de frutos de umbuzeiro (Spondias tuberosa) colhidos em quatro estádios de maturação. Bioscience Journal (Uberlândia), v. 20, n. 2, p. 65-71, 2004.

COUTO, M. A.; CANNIATTI-BRAZACA, S. G. Quantificação de vitamina C e capacidade antioxidante de variedades cítricas. Ciência e Tecnologia de Alimentos, v. 30, n. 1, p. 15-19, 2010. http://dx.doi.org/10.1590/S010120612010000500003

FRANCO, G. Table of chemical composition of foods. Rio de Janeiro: Atheneu press, 1989. 230p.

FREITAS, T. A.; OSUNA, J. T. A.; RODRIGUES, A. C. Cultivation of Physalis angulata L. and Anadenanthera colubrina [(Vell.) Brenan] species of the Brazilian semi-arid. Revista Brasileira de Plantas Medicinais, v. 8, n. esp, p. 201-204, 2006.

GOERING, H. K.; VAN SOEST, P. J. Forage fiber analysis (Apparatus, reagents, procedures and some applications). Washington, DC: USDA, 1970. 20p.

GOULARTE, V. D. S.; ANTUNES, E. C.; ANTUNES, P. L. Qualidade de maçã fuji osmoticamente concentrada e desidratada. Ciência e Tecnologia de Alimentos, v. 20, n. 2, p. 160-163, 2000. http://dx.doi.org/10.1590/S010120612000000200006

GOUVEIA, J. P. G.; AlMEIDA, F. A. C.; MEDEIROS, B. G. S.; RIBEIRO, C. F. A.; DUARTE, S. M. A. Determinação de características físico-químicas da goiaba: goiabeiras adubadas no semi-árido da Paraíba. Revista Brasileira de Produtos Agroindustriais, v. 6, n.1, p. 35-38, 2004.

GRANADA, G.G. ZAMBIAZI, R. C.; MENDONÇA, C. R. B. Abacaxi: produção, mercado e subprodutos. Boletim do CEPPA, v. 22, n. 1, p. 405-422, 2004.

HARBORNE, J. B.; WILLIAMS, C. A. Advances in flavonoid research since 1992. Phytochemistry, v. 55, n. 6, p. 481-504, 2000. http://dx.doi.org/10.1016/S0031-9422(00)00235-1 
KIM, D. O.; JEONG, S. W.; LEE, C. Y. Antioxidant capacity of phenolic phytochemicals from various cultivars of plums. Food Chemistry, v. 81, n. 2, p. 231-326, 2003.

KISSMANN, K. G., GROTH, D. Plantas infestantes e nocivas, Editora: BASF, 1995. Tomo III, p.485-487.

KUSKOSKI, E. M.; ASUERO, A. G.; MORALES, M. T.; FETT, R. Wild fruits and pulps of frozen fruits: antioxidant activity, polyphenols and anthocyanins. Ciência Rural, v. 36, n. $4 . \quad$ p. 1283-1287, 2006. http://dx.doi.org/10.1590/S0103-84782006000400037

KUSKOSKI, E. M.; ASUERO, A. G.; TRONCO, A. M.; MANCINI-FILHO, J.; FETT, R. Aplicación de diversos métodos químicos para determinar actividad antioxidante en pulpa de frutos. Ciência e Tecnologia de Alimentos, v. 25, n. 4, p. 726-732, 2005. http://dx.doi.org/10.1590/S0101-20612005000400016

LACZKÓ-ZÖLD, E.; ZUPKÓ, I.; RÉTHY, B.; CSEDO, K.; HOHMANN, J. Antioxidant activity of the fruits and hydrophilic compounds of Physalis alkekengi. Acta pharmaceutica Hungarica, v. 79, n. 2, p. 169-173. 2009.

LAGO, E. S.; GOMES, E.; SILVA, R. Produção de geléia de jambolão (Syzygium cumini Lamarck): processamento, parâmetros físico - químicos e avaliação sensorial. Ciência e Tecnologia de Alimentos. v. 26, p. 847-852. 2006. http://dx.doi.org/10.1590/S0101-20612006000400021

LANE, J. H.; EYNON, L. Determination of reducing sugars by Fehling's solution with methylene blue indicator, Norma Rodge, London, 8p, 1934.

LIMA, V. L. A. G.; MÉLO, E. A.; LIMA, D. E. S. Fenólicos e carotenóides totais em pitanga. Scientia Agricola, v. 59, n. 3, p. 447-450, 2002.

LIMA, V. L. A. G.; MÉLO, E. A.; LIMA, D. E. S. Efeito da luz e da temperatura de congelamento sobre a estabilidade das antocianinas da pitanga roxa. Ciência e Tecnologia de Alimentos, v. 25, n. 1, p. 92-94, 2005. http://dx.doi.org/10.1590/S0103-90162002000300006

LORENZI, H. Plantas daninhas do Brasil. Nova Odessa, São Paulo, Brasil, 1982. 372p.

MAIA, G.A. et al. Tecnologia em processamento de sucos e polpas tropicais. Brasília: editado pela ABEAS, 1998. $104 \mathrm{p}$.

MARINOVA, D.; RIBAROVA, F. HPLC determination of carotenoids in Bulgarian berries. Journal of Food Composition and Analysis. p. 370-374; 2006.

MATTIETTO, R. A. Influência da concentração de substrato na obtenção de vinho de acerola (Malpighia punicifolia L.). 1999. 70f. Trabalho de conclusão do curso de especialização em Tecnologia de Alimentos. Departamento de Engenharia Química, Universidade Federal do Pará.

MATTIETTO, R. A.; PONTES. M. A. N. Estudo da acerola (Malpighia punicifolia L.): Caracterização do fruto e aproveitamenoto tecnológico e obtenção de compota. XXXVII Congresso Brasileiro de Química, Natal - RN, 1997.p.251.

PANTOJA, L.; PINTO, N. A. V. D.; LOPES, C.; GANDRA, R.; SANTOS. A. S. Caracterização física e físico-química de frutos de duas variedades de tamarilho oriundas do Norte de Minas Gerais. Revista Brasileira de Fruticultura, v. 31, n. 3, p. 916-919, 2009. http://dx.doi.org/10.1590/S0100-29452009000300041

REINHARDT, D. H. ; MEDINA, V. M.; CALDAS, R. C.; CUNHA, G. A. P. ; ESTEVAM, R. F. H. Quality gradients in 'Pérola' pineapple in function of fruit size and maturation stage. Revista Brasileira Fruticultura, v. 26, n. 3, p. 544546. 2004. http://dx.doi.org/10.1590/S0100-29452004000300041

ROCHA, D. A.; ABREU, C. M. P. ; CORRÊA, A. D.; SANTOS, C. D.; FONSECA, E. W. N. Análise comparativa de nutrientes funcionais em morangos de diferentes cultivares da região de Lavras-Mg. Revista Brasileira de Fruticultura,v. 30, n. 4, P. 1124-1128, 2008. http://dx.doi.org/10.1590/S0100-29452008000400046

ROCKENBACH, I. I.; RODRIGUES, E.; CATANEO, C.; GONZAGA, L. V.; LIMA, A.; MANCINI-FILHO, J.; FETT, R. Ácidos fenólicos e atividade antioxidante em fruto de Physalis peruviana 1. Alimentos e Nutrição (Araraquara), v. 19, n. 03, p. 271-276, 2008. 
RODRIGUEZ-AMAYA, D. B. A guide to carotenoids analysis in foods. Washington: ILSI Press, 1999. 64p.

SANTANA, L. R. R.; MATSUURA, F. C. A. U.; CARDOSO, R. L. Genótipos melhorados de mamão (Carica papaya L.): avaliação sensorial e físico-química dos frutos. Ciência e Tecnologia de Alimentos, v. 24, n. 02, p. $217-222$, 2004. http://dx.doi.org/10.1590/S0101-20612004000200010

SARZI, B.; DURINGAN, J. F. Physical and chemical study of minimally processed products of 'pérola' pineapples. Revista Brasileira de Fruticultura, v. 24, n. 2, p. 333-337. 2002. http://dx.doi.org/10.1590/S010029452002000200012

SHAHIDI, F. Antioxidants: extraction, identification, application and efficacy measurement. Electronic journal of environmental, agricultural and food chemistry, v. 7, p.3325-3330, 2008

SILVA, E. M., ROGEZ, H., LARONDELLE, Y. Optimization of extraction of phenolics from Inga edulis leaves using response surface methodology. Separation and Purification Technology, v. 55. n. 14. p. 381-387. London, 2007.

SINGLETON, V. L.; ORTHOFER, R. ; LAMUELA-RAVENTÓS, R. L. Analysis of Total Phenols and Other Oxidation Substrates and Antioxidants by Means of Folin-Ciocalteu Reagent. Methods in Enzymology, v.299, p.152178, 1999. http://dx.doi.org/10.1016/S0076-6879(99)99017-1

SUN, J., CHU, Y. F., WU, X., LIU, R. H. Antioxidant and antiproliferative activities of common fruits. Journal of Agricultural and Food Chemistry, v. 50, p.7449-7454, 2002. http://dx.doi.org/10.1021/jf0207530

YEN, G. C.; DUH, P. D. Scavenging effect of methanolic extracts of peanut hulls on free-radical and active-oxygen species. Journal of Agricultural and Food Chemistry., v. 42, n. 3, p. 629-632, 1994. http://dx.doi.org/10.1021/jf00039a005

WU, S. J.; TSAIB, J. Y. ; CHANG, S. P.; LINC, D. L. ; WANG, S. S.; HUANG, S. N.; NG, L. T. Supercritical carbon dioxide extracts exhibits enhanced antioxidant and anti-inflamatory activities of Physalis peruviana. Journal of Ethnopharmacology, v. 108, n. 03, p.407-413, 2006. http://dx.doi.org/10.1016/j.jep.2006.05.027

Submetido em 19 jan. 2011; Aceito para publicação em 29 dez. 2011. 